\title{
Prediction of Relapse by Plasma Lipoprotein(a) Concentration in Children with Steroid-Sensitive Nephrotic Syndrome
}

\author{
Yukihiko Kawasaki J unzo Suzuki Ruriko Nozawa Shigeo Suzuki \\ Hitoshi Suzuki \\ Department of Pediatrics, Fukushima Medical University School of Medicine, Fukushima, J apan
}

\section{Key Words}

Lipoprotein(a) · Steroid-sensitive nephrotic syndrome • Children

\begin{abstract}
Aim: To clarify whether plasma lipoproteins, including $\mathrm{Lp}(\mathrm{a})$, can predict relapse pattern in the first years after diagnosis of nephrotic syndrome (NS), we evaluated them in patients with steroid-sensitive NS. Methods: We analyzed the medical records of 35 patients with steroidsensitive NS who were seen by us from J anuary 1992 to December 1999 followed for at least 1 year. These patients were divided into two groups. Group 1 consisted of 20 patients who infrequently relapse (IR: $<2$ in 6 months or $<3$ in a year), group 2 consisted of 15 patients who frequently relapse (FR: $\geqslant 2$ in 6 months or $\geqslant 3$ in a year). Clinical and laboratory findings such as age at onset, gender, urinalysis, serum level of total protein, albumin, and concentrations of serum lipid including lipoprotein(a) (Lp(a)) were investigated between group 1 and group 2. Results: The concentration of plasma Lp(a) in group 2 was higher than that in group 1 (81.0 \pm 35.2 vs. $35.9 \pm 26.5 \mathrm{mg} / \mathrm{dl}, \mathrm{p}<0.01$ ). On multivariate analysis using logistic regression model, the concentration of plasma Lp(a) was an independent risk factor for relapse
\end{abstract}

\section{KARGER}

Fax +41613061234 E-Mail karger@karger.ch www. karger.com

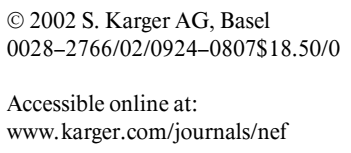

of NS. Conclusions: Our findings suggest that of all the laboratory data examined, high values of Lp(a) can predict future relapse of NS and should be well documented.

Copyright $\odot 2002$ S. Karger AG, Base

\section{Introduction}

Approximately $90 \%$ of children with minimal-change nephrotic syndrome (MCNS) will initially respond to daily prednisone with resolution of proteinuria. However, at present more than half of children with the diagnosis of nephrotic syndrome (NS) will suffer relapse. For management of patients with frequent NS, it is important to determine factors predicting relapse.

$\mathrm{Lp}(\mathrm{a})$ has been identified as an independent, inherited risk factor for atherosclerotic cardiovascular disease, and high $\mathrm{Lp}(\mathrm{a})$ concentrations have been found in patients with end-stage renal disease, whereas after kidney transplantation $\mathrm{Lp}$ (a) concentrations appear to decrease $[1,2]$. These findings suggest that the kidney may play a role in $\mathrm{Lp}$ (a) metabolism as a catabolic site or by producing factors affecting Lp(a) liver synthesis. High Lp(a) levels have been reported in patients with NS, and Lp(a) is useful for monitoring NS in children, particularly for detecting com- 
plications [3]. It has also been reported that $\mathrm{Lp}(\mathrm{a})$ is associated with metabolic abnormalities, such as fibrosis and coagulation disorder.

Therefore, to clarify whether plasma lipoproteins, including $\mathrm{Lp}(\mathrm{a})$, can predict a relapse pattern, we evaluated them in patients with steroid-sensitive NS.

\section{Patients and Methods}

\section{Study Characteristics}

This study was conducted as a retrospective chart review of all pediatric patients with steroid-sensitive NS referred to and followed by the pediatric nephrologists at Fukushima Medical University from January 1992 to December 1999, for whom at least 12 months of follow-up were completed. NS was defined as heavy proteinuria of more than $0.1 \mathrm{~g} / \mathrm{kg}$ per day, low serum albumin levels (less than $2.5 \mathrm{~g} / \mathrm{dl}$ in children under 6 years and less than $3.0 \mathrm{~g} / \mathrm{dl}$ in older children), and/or obvious edema. Patients with NS infrequently relapse (IR: $<2$ times in 6 months or $<3$ in a year) or frequently relapse (FR: $\geqslant 2$ times in 6 months or $\geqslant 3$ in a year). All patients were treated with standard therapy. Standard therapy consisted of prednisolone at a dose of $2 \mathrm{mg} / \mathrm{kg}$ per day for 4 weeks, followed by $2 \mathrm{mg} / \mathrm{kg}$ on alternate days for 4 weeks. Thirty-five patients meeting the above criteria were divided into two groups. Group 1 consisted of 20 patients with infrequently relapsing NS (IRNS), and group 2 consisted of 15 patients with frequently relapsing NS (FRNS). Ten healthy children served as controls. The medical records of patients diagnosed with NS were analyzed and information at initial presentation was noted [age at onset, gender, hematuria (presence of at least 5 red blood cells per high power field), and proteinuria] along with laboratory findings [serum total protein, albumin, proteinuria, BUN, creatinine, and lipid (total cholesterol, triglyceride, high-density lipoprotein (HDL)-cholesterol, chylomicron, low-density lipoprotein (LDL), very-low-density lipoprotein (VLDL), lipoprotein(a) (Lp(a)) and apoprotein (apo)A1, apoA2, apoB, apoC2, apoC3, apoE)]. These initial characteristics at presentation and laboratory findings in the groups were compared.

\section{Laboratory Procedures}

The serum samples for laboratory examination were collected at onset (days $1.7 \pm 1.4$ after admission) prior to treatment and stored at $-20^{\circ} \mathrm{C}$. Serum total protein and albumin, proteinuria, BUN, creatinine, total cholesterol, triglyceride, and HDL cholesterol were measured according to standard procedures. ApoA I, apoA II, apoB, apoC II, apoC III and apoE were measured by the turbidimetric immunoassay method. Lp(a) was measured using a commercial enzyme-linked immunosorbent assay method. Chylomicrons, LDL, and VLDL were measured by turbidimetry.

\section{Statistics}

Data are expressed as mean values \pm SD. Statistical analysis was performed on a Macintosh computer with a software package for statistical analysis (Stat View, Abacus Concepts, Berkeley, Calif., USA). Differences of laboratory findings between both groups were assessed by Mann-Whitney's rank sum. We evaluated the potential factors for relapse of NS using the logistic regression model for a multivariate analysis. Evaluation of correlation was determined with Fisher's $r$ test. $\mathrm{p}<0.05$ was considered significant.

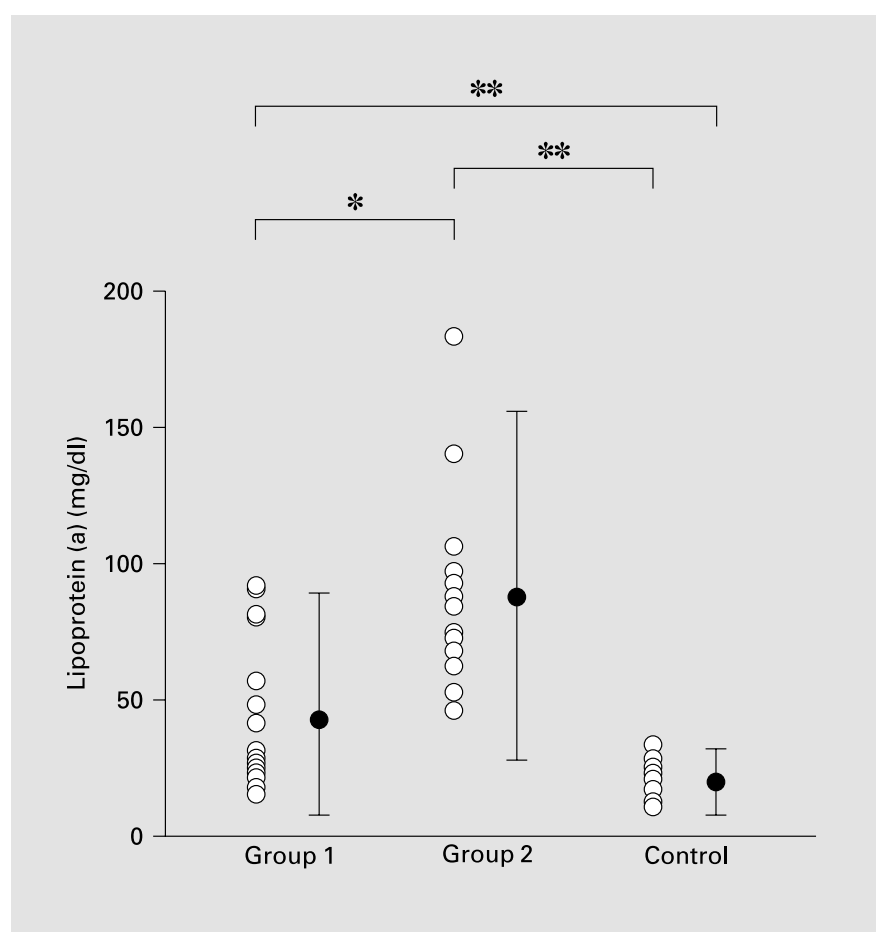

Fig. 1. Comparison of lipoprotein(a) among three groups. ${ }^{*} \mathrm{p}<0.01$, $* * \mathrm{p}<0.001$.

Table 1. Comparison of baseline characteristics, urinalysis and laboratory data between groups 1 and 2

\begin{tabular}{lccc}
\hline & $\begin{array}{l}\text { Group 1 } \\
(\mathrm{n}=20)\end{array}$ & $\begin{array}{l}\text { Group 2 } \\
(\mathrm{n}=15)\end{array}$ & $\mathrm{p}$ \\
\hline Mean age, years & $7.9 \pm 4.4$ & $6.2 \pm 3.5$ & n.s. \\
Male-to-female ratio & $13: 7$ & $10: 5$ & n.s. \\
$\begin{array}{l}\text { Periods from onset to } \\
\quad \text { admission, days }\end{array}$ & $8.5 \pm 6.5$ & $11.0 \pm 4.0$ & n.s. \\
\hline Urinalysis & & & \\
$\quad$ Proteinuria, g/day & $4.6 \pm 4.5$ & $6.0 \pm 7.1$ & n.s. \\
$\quad$ Hematuria & $3 / 20(15.0 \%)$ & $2 / 15(13.3 \%)$ & n.s. \\
\hline Laboratory data & & & \\
$\quad$ BUN, mg/dl & $12.3 \pm 10.4$ & $20.4 \pm 27.0$ & n.s. \\
$\quad$ Creatinine, mg/dl & $0.49 \pm 0.19$ & $0.46 \pm 0.26$ & n.s. \\
$\quad$ Total protein, g/dl & $4.9 \pm 1.1$ & $4.4 \pm 1.0$ & n.s. \\
$\quad$ Albumin, g/dl & $2.5 \pm 1.1$ & $2.0 \pm 0.7$ & n.s. \\
\hline
\end{tabular}

n.s. = Not significant.

Mann-Whitney' rank sum test, Fisher's $r$ test. 
Table 2. Comparison of lipids among the three groups

\begin{tabular}{lccc}
\hline & $\begin{array}{l}\text { Control group } \\
(\mathrm{n}=10)\end{array}$ & $\begin{array}{l}\text { Group 1 } \\
(\mathrm{n}=20)\end{array}$ & $\begin{array}{l}\text { Group 2 } \\
(\mathrm{n}=15)\end{array}$ \\
\hline Total cholesterol, mg/dl & $138.7 \pm 35.1$ & $390.7 \pm 189.1$ & $480.3 \pm 140.9$ \\
Triglyceride, mg/dl & $79.0 \pm 23.3$ & $185.4 \pm 109.8$ & $280.2 \pm 168.6$ \\
HDL-cholesterol, mg/dl & $45.4 \pm 21.7$ & $86.1 \pm 41.0$ & $78.9 \pm 28.9$ \\
Chylomicron, mg/dl & $66.3 \pm 31.6$ & $160.5 \pm 195.9$ & $139.7 \pm 125.2$ \\
LDL, mg/dl & $360.8 \pm 75.7$ & $1,362.4 \pm 810.4$ & $1,810.0 \pm 898.7$ \\
VLDL, mg/dl & $35.5 \pm 12.7$ & $191.0 \pm 147.9$ & $345.5 \pm 273.6$ \\
Lipoprotein(a), mg/dl & $11.5 \pm 5.8^{*}$ & $35.9 \pm 26.5^{*}$ & $81.0 \pm 35.2^{*}$ \\
Apoprotein-A1, mg/dl & $113.9 \pm 28.2$ & $172.0 \pm 43.9$ & $176.6 \pm 32.8$ \\
Apoprotein-A2, mg/dl & $26.0 \pm 7.2$ & $35.9 \pm 11.2$ & $40.2 \pm 9.5$ \\
Apoprotein-B, mg/dl & $64.3 \pm 13.5$ & $157.8 \pm 59.6$ & $185.0 \pm 32.8$ \\
Apoprotein-C2, mg/dl & $3.2 \pm 0.8$ & $7.8 \pm 3.1$ & $9.5 \pm 3.7$ \\
Apoprotein-C3, mg/dl & $6.1 \pm 2.1$ & $13.6 \pm 6.2$ & $19.9 \pm 12.1$ \\
Apoprotein E, mg/dl & $3.5 \pm 0.9$ & $7.5 \pm 3.2$ & $9.6 \pm 3.0$ \\
\hline
\end{tabular}

${ }^{*} \mathrm{p}<0.05$, Mann-Whitney’ rank-sum test.
Table 3. A multivariate analysis of the risk factors for relapse of NS

\begin{tabular}{lll}
\hline Risk factor & \multicolumn{2}{l}{ Odds ratio $\mathrm{p}$} \\
\hline Boys & 1.05 & 0.87 \\
Age at onset, years & 0.98 & 0.93 \\
Proteinuria, g/day & 1.19 & 0.14 \\
Creatinine, mg/dl & 0.93 & 0.15 \\
Albumin, g/dl & 0.73 & 0.91 \\
Total cholesterol, mg/dl & 0.99 & 0.65 \\
Triglyceride, mg/dl & 0.98 & 0.41 \\
HDL-cholesterol, mg/dl & 1.01 & 0.72 \\
Chylomicron, mg/dl & 0.97 & 0.91 \\
LDL, mg/dl & 1.22 & 0.36 \\
VLDL, mg/dl & 0.89 & 0.32 \\
Lipoprotein(a), mg/dl & 2.91 & 0.03 \\
Apoprotein-A1, mg/dl & 0.94 & 0.13 \\
Apoprotein-A2, mg/dl & 1.21 & 0.37 \\
Apoprotein-B, mg/dl & 0.98 & 0.91 \\
Apoprotein-C2, mg/dl & 0.91 & 0.83 \\
Apoprotein-C3, mg/dl & 1.01 & 0.94 \\
Apoprotein E, mg/dl & 1.48 & 0.18 \\
\hline
\end{tabular}

\section{Results}

(1) Comparison of baseline characteristics among group 1, group 2 and control group, and of urinalysis and laboratory data between group 1 and group 2 (table 1).

Age at onset, male-to-female ratio, and the period from onset to admission did not differ among the groups. Pro- teinuria and presence or absence of hematuria did not differ between the two groups. In addition, laboratory data such as, BUN, Cr level, concentration of total protein and albumin did not differ between the two groups.

(2) Comparison of serum lipid among groups 1,2 and control (tables 2, 3; fig. 1).

Each concentration of serum lipids including total cholesterol, triglyceride, HDL-cholesterol, chylomicron, LDL, VLDL, Lp(a), and apoA1, apoA2, apoB, apoC2, apoC3, apoE was higher in group 1 and group 2 than in the control group.

The concentration of serum $\mathrm{Lp}(\mathrm{a})$ in group 2 was higher than that in group $1(81.0 \pm 35.2$ vs. $35.9 \pm 26.5 \mathrm{mg} /$ $\mathrm{dl}, \mathrm{p}<0.01)$. Mean values $+2 \mathrm{SD}$ of plasma $\mathrm{Lp}(\mathrm{a})$ in the control group was $23.1 \mathrm{mg} / \mathrm{dl}$. Seven (35.0\%) patients in group 1 and $15(100 \%)$ in group 2 had plasma $L p(a)$ concentrations higher than $23.1 \mathrm{mg} / \mathrm{dl}$, which is the upper limit of normal.

On multivariate analysis using logistic regression mod$\mathrm{el}$, the concentration of plasma Lp(a) was an independent risk factor for relapse of NS.

Concentrations of the other serum lipids including total cholesterol, triglyceride, HDL-cholesterol,chylomicron, LDL, VLDL, and apoA1, apoA2, apoB, apoC2, apoC3, apoE were not independent risk factors for relapse of NS.

(3) Relationships between $\mathrm{Lp}$ (a) concentration and other laboratory data (table 4).

Plasma Lp(a) concentrations were positively correlated with other serum lipids including total cholesterol, triglyc- 
Table 4. Relationships between Lp(a) concentration and other laboratory data

\begin{tabular}{lcc}
\hline & $\mathrm{r}$ & $\mathrm{p}$ \\
\hline Total cholesterol & 0.75 & $<0.001$ \\
Triglyceride & 0.45 & $<0.001$ \\
HDL cholesterol & 0.24 & n.s. \\
Apo A1 & 0.49 & 0.003 \\
Apo A2 & 0.53 & 0.001 \\
Apo B & 0.53 & $<0.01$ \\
LDL & 0.72 & $<0.001$ \\
\hline VLDL & 0.72 & $<0.001$ \\
Chylomicron & 0.28 & n.s. \\
Apo C2 & 0.64 & $<0.001$ \\
Apo C3 & 0.65 & $<0.001$ \\
Apo E & 0.66 & $<0.001$ \\
Serum total protein & -0.098 & n.s. \\
Serum albumin & -0.51 & 0.03 \\
\hline
\end{tabular}

n.s. $=$ Not significant .

eride, LDL, VLDL, and apoA1, apoA2, apoB, apoC2, apoC3, apoE, and negatively correlated serum albumin level. Plasma $\mathrm{Lp}$ (a) concentrations were not associated with proteinuria and fibrinogen levels $(r=-0.12 \mathrm{p}=0.59$, $\mathrm{r}=0.30 \mathrm{p}<0.10$, respectively).

(4) Relationship between $\mathrm{Lp}$ (a) concentration and frequency of relapse.

Plasma Lp(a) concentration was unrelated to the frequency of relapse.

\section{Discussion}

This study found that concentration of plasma $L p(a)$ in patients with FRNS was higher than that in patients with IRNS, and that this concentration may serve as a marker for prediction of FRNS. Previously, some studies examined prediction of relapse of NS [4-6]. Although Cornfield et al. [4], reported that the older the patient at onset, the worse the prognosis, most of their patients had glomerulonephritis, a histopathological finding associated with poor response to steroids. In addition, Siegel et al. [5] found no statistically significant relationship between relapses and age at onset, gender, or hematuria. Takeda et al. [7] reported that a low serum level of total protein and young age at onset increased relapse rate.

However, no study has examined the relationship between plasma $\mathrm{Lp}(\mathrm{a})$ and prediction of FRNS. Our study is unique in investigating the likelihood of being a patient with FRNS based on serum Lp(a) concentration.

$\mathrm{Lp}(\mathrm{a})$ is a plasma lipoprotein consisting of a low-density lipoprotein (LDL) particle linked by a disulfide bond to a highly polymorphic glycoprotein, apoprotein(a) [8]. Recently, several studies $[9,10]$ have shown that $\operatorname{Lp}(a)$ is an independent risk factor in atherosclerosis and thrombosis. In addition, studies investigating lipoprotein abnormalities in renal diseases with nephrosis have found a consistent increase in plasma $\mathrm{Lp}(\mathrm{a})$ that parallels increases in cholesterol, triglyceride, and apoB [11-13].

In our study, serum concentrations of serum lipids including total cholesterol, triglyceride, HDL-cholesterol, chylomicron, LDL, VLDL, Lp(a) and apoA1, apoA2, apoB, apoC2, apoC3, and apoE in patients with NS were higher than those in the control group. In addition, plasma $L p(a)$ concentrations were positively correlated with other serum lipids including total cholesterol, triglyceride, LDL, VLDL, and apoA1, apoA2, apoB, apoC2, apoC3, and apoE, and were negatively correlated serum albumin level. Additionally, on multivariate analysis using logistic regression model, the concentration of plasma $\mathrm{Lp}$ (a) was an independent risk factor for relapse of NS.

The only factor found to be associated with future relapse is high serum $L p(a)$ level.

The reasons for high $\operatorname{Lp}(\mathrm{a})$ level in patients with steroid-sensitive NS have been speculated to include the following [15]: (1) enhancement of hepatic synthesis of Lp(a) may be stimulated by decreased serum albumin concentration, and (2) large particle Lp(a) is increased in NS due to a size selectivity effect. In our study, plasma Lp(a) level was negatively correlated with serum albumin concentration, but was not related to urinary protein excretion. Therefore, it is possible that high values of $L p(a)$ in patients with NS were due to the former reason.

However, the mechanism why plasma Lp(a) level in FRNS was higher than that in IRNS remains unclear.

Recently, many studies have shown participation of Lp(a) in metabolic abnormalities, e.g. fibrosis and coagulation disorder [14-16]. It has been proposed that $\mathrm{Lp}(\mathrm{a})$ may inhibit the binding of plasminogen to cells and fibrin by a competitive mechanism and thereby interfere with the fibrinolytic process [15] and accelerate the coagulation activity in the kidney. Soulat et al. [15] reported that the increased binding of $\mathrm{Lp}(\mathrm{a})$ to fibrin and cell surfaces observed during a flare-up of nephritic syndrome was significantly correlated with the apo(a)/plasminogen molar concentration ratio, indicating that it was directly related to the concentration of $\mathrm{Lp}(\mathrm{a})$ and inversely proportional to the level of plasminogen. 
These findings might suggest that high values of plasma $L p(a)$ indicate differences in degeneration and coagulation in glomeruli between FRNS and IRNS. The values of plasminogen and fibrin were not measured at onset in our study. Further investigations concerning coagulants, such as plasminogen and fibrin in patients with IRNS and FRNA are necessary.

One of the most difficult problems in the care of children with NS remains the occurrence of relapse, despite initial response to steroid. These patients with FRNS are at high risk of complications of steroid and/or cytotoxic agents. If predictive factors are established, efforts can be directed toward minimizing these complications. High value of $L p(a)$ identified in our study may be helpful in this regard. These findings suggest that $\mathrm{Lp}$ (a) may play an important role in the pathogenesis of FRNS.

A high $\mathrm{Lp}(\mathrm{a})$ level would alert physicians caring for these patients to monitor them closer and allow them to appropriately counsel patients and their caretakers.

In conclusion, our findings suggest that of all the laboratory data examined, high values of $L p(a)$ can predict future relapse of NS and should be well documented.

\section{References}

1 Haffner SM, Gruber KK, Aldrete G, Morales PA, Stern MP, Tuttle KR: Increased Lipoprotein(a) in chronic renal failure. J Am Soc Nephrol 1992;3:1156-1162.

2 Barbagallo CM, Averna MR, Sparacino V, GalioneA, Capoto F, Scafidi V, Amato S, Mancino C, Cefalu AB, Notarbartolo A: Lipoprotein(a) levels in end-stage renal failure and renal transplantation. Nephron 1993;64:560-564.

3 Garnotel R, Roussel B, Pennaforte F, Randoux A, Gillery P: Changes in serum lipoprotein(a) levels in children with corticosensitive nephritic syndrome. Pediatr Nephrol 1996;10:699_ 701.

4 Confield D, Schwartz MW: Nephrosis: A long study of children treated with corticosteroids. J Pediatr 1966;68:507-515.

5 Siegel NJ, Goldberg B, Krassner LS, Hayslett JP: Long-term follow-up of children with steroid responsive nephrotic syndrome. J Pediatr 1972;81:251-258
6 International Study of Kidney Diseases in Children: Nephrotic syndrome in children: Prediction of histopathology from clinical and laboratory characteristics at time of diagnosis. Kidney Int 1978;13:159-165.

7 Takeda A, Matsutani H, Niimura F, Ohgushi $\mathrm{H}$ : Risk factors for relapse in childhood nephrotic syndrome. Pediatr Nephrol 1996;10: 740-741.

8 Utermann G: The mysteries of lipoprotein(a). Science 1989;246:904-910.

9 MBewu AD, Durrington PN: Lipoprotein(a): Structure, properties and possible involvement in thrombogenesis and atherogenesis. Atherosclerosis 1990;85:1-14.

10 Loscalzo J: Lipoprotein(a): A unique risk factor for atherothrombotic disease. Arteriosclerosis 1990;10:672-679.

11 Muls E, Rossenneu M, Daneels Rschurgers M, Boelaert J: Lipoprotein distribution and composition in the human nephrotic syndrome. Atherosclerosis 1985;54:225-227.

12 Thabet MA, Salcedo JR, Chan JC: Hyperlipidemia in childhood nephrotic syndrome. Pediatr Nephrol 1993;7:559-666.
13 Garnotel R, Roussel B, Pennaforte F, Randoux A, Gillery P: Changes in serum lipoprotein(a) levels in children with corticosensitive nephrotic syndrome. Pediatr Nephrol 1996;10: 699-701.

14 Nakahara C, Kobayashi K, Hamaguchi H, Kanemoto K, Kashiwagi T, Matsui A: Plasma lipoprotein(a) levels in children with minimal lesion nephritic syndrome. Pediatr Nephrol 1999;13;657-661.

15 Soulat T, Loyau S, Baudouin V, Maisonneuve L, Hurtaud-Riux MF, Schlegel N, Loirat C, Angle-Cano E: Effect of individual plasma lipoprotein(a) variations in vivo on its competition with plasminogen for fibrin and cell binding. Arteriscler Tromb Vasc Biol 2000;20;575584.

16 Doucet C, Mooser V, Gonbert S, Raymond F, Chapman J, Thillet J: Plasma lipoprotein(a) in the nephrotic of lipoprotein(a) and apolipoprotein(a) in plasma and urine. JASN 2000: 11;13;507-513. 